Int. J. Morphol.,

39(3):705-709, 2021.

\title{
Importancia del Sitio de Terminación de la Vena Cefálica del Antebrazo
}

\author{
Importance of the Cephalic Antebrachial Vein Termination Site
}

Edgar Giovanni Corzo Gómez ${ }^{1}$; Diana Isabel Cáceres Rivera²; Rocío Calderón Mortigo ${ }^{3}$ \& Luis Alberto López Romero ${ }^{4}$

CORZO, G. E. G.; CÁCERES, R. D. I.; CALDERÓN, M. R. \& LÓPEZ, R. L. A. Importancia del sitio de terminación de la vena cefálica del antebrazo. Int. J. Morphol., 39(3):705-709, 2021.

RESUMEN: Las venas superficiales de la fosa cubital se han estudiado con varios fines, venopunciones, cateterismos, fístulas, entre otros. Se han descrito varios patrones venosos en esta región en diferentes poblaciones. En uno de estos la vena cefálica termina en la vena basílica a nivel del antebrazo generando posibles riesgos de lesiones durante la canalización. En el presente estudio se evaluaron 200 miembros superiores de 100 personas colombianas, 50 mujeres y 50 hombres, 70 de ellas nacidas en la ciudad de Bucaramanga y 30 en la ciudad de Villavicencio. Se registró el número de venas cefálicas y su sitio de terminación, en el brazo o antebrazo. El patrón venoso que predominó en ambos miembros superiores fue el tipo I. El patrón IV en el cual la vena cefálica termina en la vena basílica se presentó en 12 casos $(6 \%)$ en el miembro superior derecho y en 17 casos $(8,5 \%)$ en el miembro superior izquierdo. Se encontró dos venas cefálicas entre el $13 \%$ y 13,5\% dependiendo del lado y tres venas cefálicas entre el $1 \%$ y $2 \%$. Conocer esta particularidad de los patrones venosos podría disminuir el riesgo de complicaciones durante la venopunción.

PALABRAS CLAVE: Vena cefálica del antebrazo. Venopunción. Patrones venosos.

\section{INTRODUCCIÓN}

La punción de las venas superficiales del miembro superior es un procedimiento necesario y frecuente, pero puede generar riesgos durante su ejecución, que pueden tener origen en la técnica misma, en la anatomía de las venas o en las variaciones anatómicas de las estructuras vasculares y nerviosas circundantes, entre otras causas. Boeson et al. (2000) señalaron la posibilidad de lesión de nervios cercanos con consecuentes lesiones motoras y sensitivas. Horowitz (2001) enfatizó en el riesgo de lesión de los nervios cutáneos antebraquiales, y de la rama superficial del nervio radial. Lirk et al. (2004) señalaron el riesgo de lesionar una arteria cercana o de aplicación equivocada de una sustancia por una arteria. Barria et al. (2006) denotaron como la punción venosa es más difícil en pacientes obesos y niños con abundante grasa bajo la piel. Otros autores han advertido como los cambios en el lugar de punción afectan más a los niños, Racadio et al. (2001). En este sentido, los estudios anatómicos han tomado mayor importancia en la actualidad, dado que la afectación de una vena durante su pun- ción o de una estructura vecina, puede conllevar a un mayor riesgo de complicaciones.

Los estudios de del Sol et al. (1988) en niños y de del Sol et al. (2007) en el grupo étnico Mapuche en Chile generaron una clasificaciónde los patrones venosos que se aplica actualmente en varios países. Se describe que, en el patrón Tipo I: La vena cefálica (VC) se divide en vena intermedia basílica (VIB) y vena intermedia cefálica (VIC), uniéndose a la vena basílica (VB) y vena cefálica accesoria (VCA) respectivamente. En el patrón Tipo II: la VC origina la vena intermedia del codo (VICo), que se une a la VB; no existe VCA, siendo la VICo de mayor calibre y la VIA drena en la VB. En el Tipo III: no existe comunicación entre la VB y la VC a nivel de la fosa cubital; la VIA suele drenar en la VB. En el Tipo IV: la VC drena en la VB y la VIA drena en la VC. A estos cuatro patrones se les ha unido la clásica presentación en "M" en donde la VIA se divide en una rama que se une a la VC y otra rama que se une a la VB. (Fig, 1).

\footnotetext{
${ }^{1}$ Médico, Magíster en Ciencias Básicas Biomédicas, Docente del programa de Enfermería, Universidad Cooperativa de Colombia, campus Bucaramanga. ${ }^{2}$ Enfermera, Magíster en Enfermería, Doctora en Biomedicina, Docente del programa de Enfermería, Universidad Cooperativa de Colombia, Campus Bucaramanga.

${ }^{3}$ Enfermera, Especialista en salud ocupacional, Maestrante en ciencias de la Enfermería, Profesora del programa de Enfermería, Universidad Cooperativa de Colombia, campus Villavicencio.

${ }^{4}$ Enfermero, Magíster en Epidemiología. Salud Mía EPS. Fundación Cardiovascular de Colombia.
} 
En población colombiana se han realizado estudios similares (Corzo Gómez et al., 2010, 2014) encontrando varias similitudes con hallazgos previos. Llama la atención el patrón tipo IV, en el cual la vena cefálica del antebrazo no sube hasta el brazo y por el contrario desemboca en la vena basílica, a nivel del antebrazo, formando una curva muy pronunciada. Se ha intentado relacionar este patrón, como una posible causa de complicaciones durante los cateterismos en niños (Corzo Gómez et al., 2016) en razón a la curva que realiza la vena cefálica formando un ángulo muy cerrado, lo cual puede generar lesiones involuntarias durante la canalización principalmente por extravasación del catéter.

En el presente estudio se describió la frecuencia de los patrones venosos y la frecuencia de presentación de venas cefálicas que terminan en la vena basílica a nivel del antebrazo en una muestra de dos ciudades de Colombia.

\section{MATERIAL Y MÉTODO}

Diseño del estudio: Estudio descriptivo en personas mayores de 18 años de ambos géneros residentes en dos ciudades de Colombia (Bucaramanga y Villavicencio). Se realizó un muestreo a conveniencia que incluyó 200 miembros superiores $50 \%$ de hombres y $50 \%$ de mujeres.
Mediciones: Se realizó la valoración de las venas superficiales realizando el siguiente procedimiento: El participante se ubicó en posición sentado con el antebrazo extendido sobre una superficie firme y plana de manera que deje visible las venas superficiales. Se aplicó un torniquete de velcro por un tiempo no superior a 20 segundos. Se visualizó la presencia de una o varias venas cefálicas y su sitio de terminación y la información y se registró en un formato escrito incluyendo un registro fotográfico. Se determinaron los siguientes elementos anatómicos en cada miembro superior: Presencia o no de una vena cefálica del antebrazo, presencia de más de una vena cefálica y determinación del número de ellas y sitio de terminación de cada una.

Análisis de datos. Se realizó un análisis descriptivo de las variables sociodemográficas y anatómicas. Se estimaron frecuencia de variables cuantitativas mediante promedios y desviación estándar.

Aspectos éticos. Esta investigación se realizó de acuerdo a la resolución No. 08430 de 1993 del Ministerio de Salud de Colombia y se consideró de "riesgo menor al mínimo"Todos los participantes del estudio firmaron consentimiento informado por escrito; adicionalmente el estudio contó con la aprobación de los comités de ética de las instituciones donde se llevó cabo el estudio y cumplió con la normatividad nacional e internacional para la investigación en seres humanos.

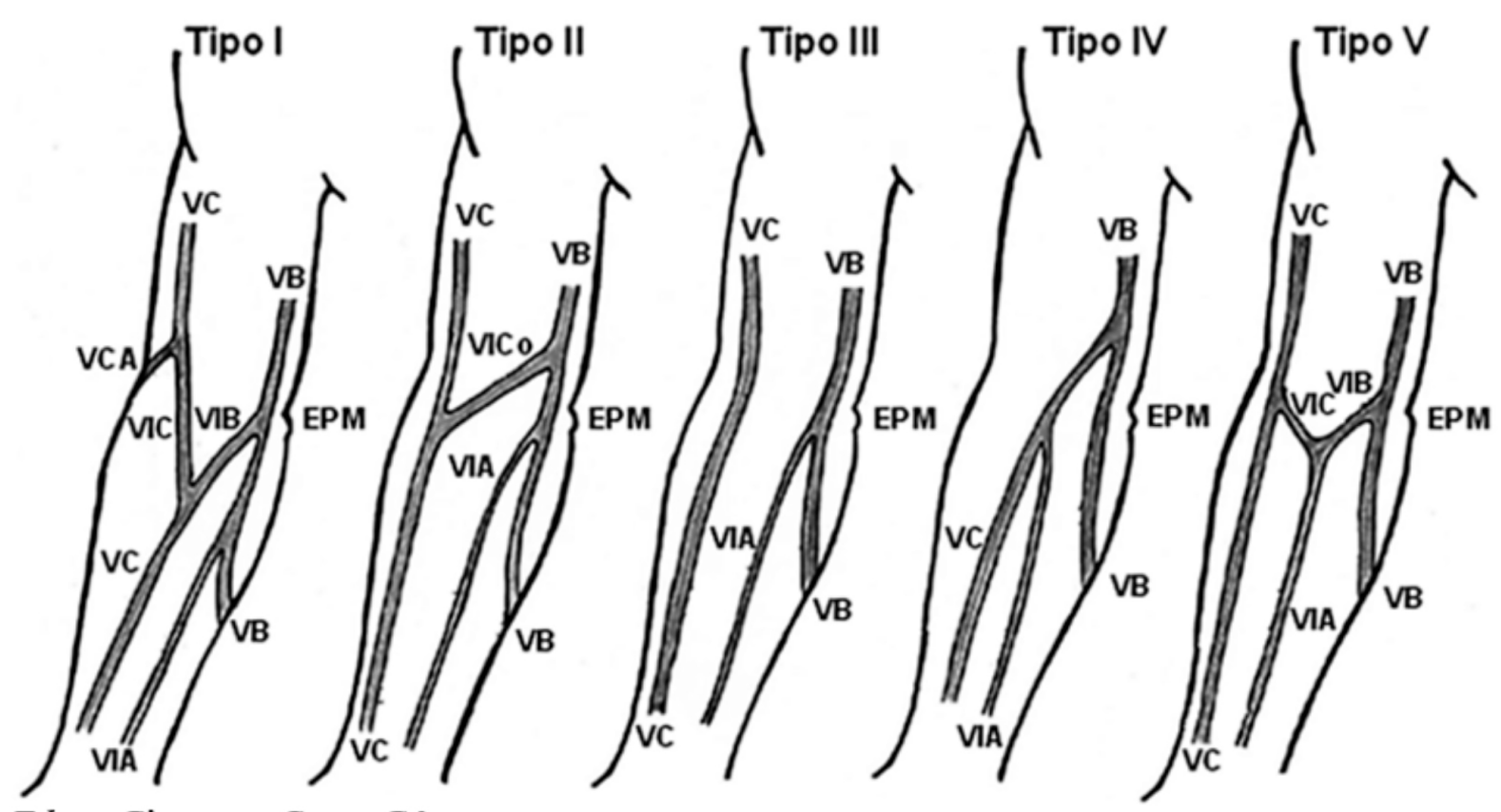

\section{Edgar Giovanny Corzo Gómez}

Fig. 1. Descripción de los patrones venosos de la fosa cubital según la propuesta de del Sol et al. (1988) y del Sol et al. (2007). VC: vena cefálica; VB: vena basílica; VCA: vena cefálica accesoria; VIC: vena intermedia cefálica; VIB: vena intermedia basílica; VIA: vena intermedia del antebrazo; VICo: vena intermedia del codo; EPM: epicóndilo medial. 


\section{RESULTADOS}

El patrón venoso que predominó en ambos miembros superiores fue el tipo I. El patrón IV en el cual la vena cefálica termina en la vena basílica a nivel del antebrazo (Fig. 2) se presentó en 12 casos (6\%) en el miembro superior derecho y en 17 casos $(8,5 \%)$ en el miembro superior izquierdo. (Tabla I).

En el miembro superior derecho se presentó una vena cefálica en 171 casos, $(85,5 \%)$, dos venas cefálicas en 27 casos $(13,5 \%)$ y tres venas cefálicas en 2 casos (1 $\%$ ), (Tabla III). En el miembro superior izquierdo se presentó una vena cefálica en 170 casos $(85 \%)$, dos venas cefálicas en 26 casos (13\%) y tres venas cefálicas en 4 casos ( $2 \%)$, (Tabla IV).
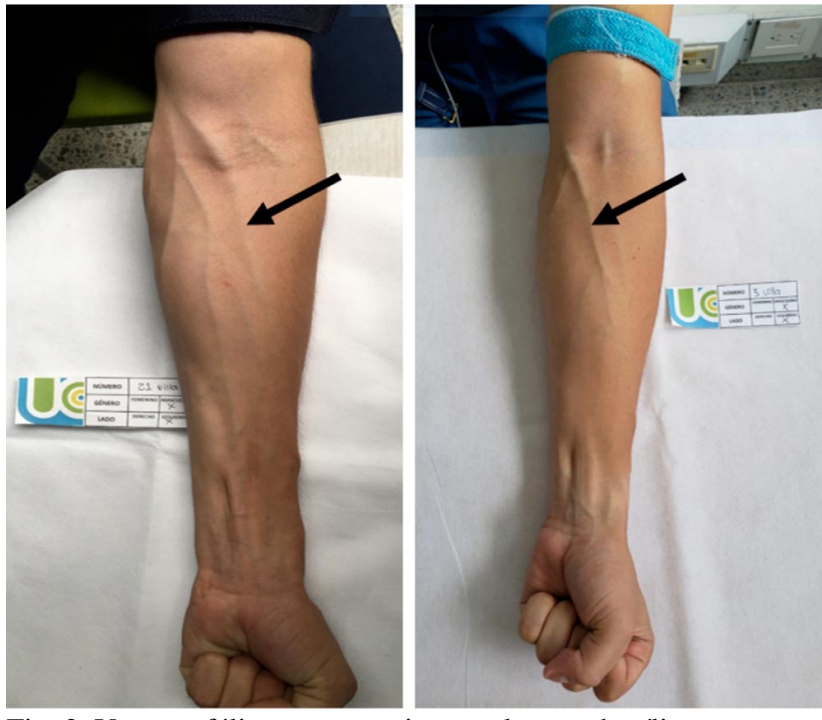

Fig. 2. Venas cefálicas que terminan en la vena basílica.

Tabla I. Distribución de frecuencia de los patrones venosos de la fosa cubital en el miembro superior derecho en una muestra de 100 personas.

\begin{tabular}{lccc}
\hline $\begin{array}{l}\text { Tipo de patrón venoso en el } \\
\text { miembro superior derecho }\end{array}$ & $\begin{array}{c}\text { Porcentaje (número) } \\
\text { Total }\end{array}$ & $\begin{array}{c}\text { Porcentaje (número) } \\
\text { Bucaramanga }\end{array}$ & $\begin{array}{c}\text { Porcentaje (número) } \\
\text { Villavicencio }\end{array}$ \\
\hline no determinado & $2,50(5)$ & $2,86(4)$ & $1,67(1)$ \\
patrón I & $41,50(83)$ & $40,00(56)$ & $45,00(27)$ \\
patrón II & $15,00(30)$ & $12,86(18)$ & $20,00(12)$ \\
patrón III & $25,00(50)$ & $28,57(40)$ & $16,67(10)$ \\
patrón IV & $6,00(12)$ & $5,71(8)$ & $6,67(4)$ \\
patrón V & $10,00(20)$ & $10,0(14)$ & $10,0(6)$ \\
\hline
\end{tabular}

Tabla II. Distribución de frecuencia de los patrones venosos de la fosa cubital en el miembro superior izquierdo en una muestra de 100 personas.

\begin{tabular}{lccc}
\hline $\begin{array}{l}\text { Tipo de patrón venoso en el } \\
\text { miembro superior izquierdo }\end{array}$ & $\begin{array}{c}\text { Porcentaje (número) } \\
\text { Total }\end{array}$ & $\begin{array}{c}\text { Porcentaje (número) } \\
\text { Bucaramanga }\end{array}$ & $\begin{array}{c}\text { Porcentaje (número) } \\
\text { Villavicencio }\end{array}$ \\
\hline no determinado & $2,00(4)$ & $1,43(2)$ & $3,33(2)$ \\
patrón I & $43,00(86)$ & $42,14(59)$ & $45,00(27)$ \\
patrón II & $14,00(28)$ & $12,86(18)$ & $16,67(10)$ \\
patrón III & $23,00(46)$ & $26,43(37)$ & $15,00(9)$ \\
patrón IV & $8,50(17)$ & $7,86(11)$ & $10,00(6)$ \\
patrón V & $9,50(19)$ & $9,29(13)$ & $10,00(6)$ \\
\hline
\end{tabular}

Tabla III. Distribución de frecuencia del número de venas cefálicas en el miembro superior derecho en una muestra de 100 personas.

\begin{tabular}{cccc}
\hline $\begin{array}{c}\text { Número de venas cefálicas en el } \\
\text { miembro superior derecho }\end{array}$ & $\begin{array}{c}\text { Porcentaje (número) } \\
\text { Total }\end{array}$ & $\begin{array}{c}\text { Porcentaje (número) } \\
\text { Bucaramanga }\end{array}$ & $\begin{array}{c}\text { Porcentaje (número) } \\
\text { Villavicencio }\end{array}$ \\
\hline 1 & $85,50(171)$ & $88,57(124)$ & $78,33(47)$ \\
2 & $13,50(27)$ & $10,0(14)$ & $21,67(13)$ \\
3 & $1,00(2)$ & $1,43(2)$ & $0,00(0)$ \\
\hline
\end{tabular}

Tabla IV. Distribución de frecuencia del número de venas cefálicas en el miembro izquierdo derecho en una muestra de 100 personas.

\begin{tabular}{cccc}
\hline $\begin{array}{l}\text { Número de venas cefálicas en el } \\
\text { miembro superior izquierdo }\end{array}$ & $\begin{array}{c}\text { Porcentaje (número) } \\
\text { Total }\end{array}$ & $\begin{array}{c}\text { Porcentaje (número) } \\
\text { Bucaramanga }\end{array}$ & $\begin{array}{c}\text { Porcentaje (número) } \\
\text { Villavicencio }\end{array}$ \\
\hline 1 & $85,00(170)$ & $88,57(124)$ & $76,67(46)$ \\
2 & $13,00(26)$ & $9,29(13)$ & $21,67(13)$ \\
3 & $2,00(4)$ & $2,14(3)$ & $1,67(1)$ \\
\hline
\end{tabular}


Cuando en el miembro superior derecho se presentó una sola vena cefálica, esta vena terminó en el antebrazo e 12 casos $(6 \%)$, cuando se presentó una segunda vena cefálica, esta vena terminó en el antebrazo en 22 casos (11 $\%)$ y cuando se presentó una tercera vena cefálica, ella terminó en el antebrazo en 2 casos (1\%) (Tabla III). Cuando en el miembro superior izquierdo se presentó una sola vena cefálica, esta vena terminó en el antebrazo en 17 casos $(8,5$ $\%)$, cuando se presentó una segunda vena cefálica, esta vena terminó en el antebrazo en 22 casos $(11 \%)$ y cuando se presentó una tercera vena cefálica, ella terminó en el antebrazo en 2 casos ( $1 \%)$. (Tabla IV).

\section{DISCUSIÓN}

En los resultados fue evidente la mayor frecuencia de presentación del patrón venoso tipo I en ambos lados, lo cual se ha observado de igual manera en estudios de otras poblaciones (AlBustami et al., 2014; Bekel et al., 2018).

El patrón IV, se encontró entre el $6 \%$ y el 8,5\% dependiendo del lado derecho e izquierdo respectivamente. Bekel et al. reportaron el patrón tipo IV en un $14 \%$.Del patrón IV hay variedad en los reportes, valores altos en población nigeriana (25\%) (Ukoha et al., 2013), bajos en población de Malasia (4,7 \%) (Dharap \& Shaharuddin, 1994) y muy bajos en población de India (0,8 \%) (Vasuda, 2013).

En estudios de población colombiana, el patrón IV se ha evidenciado en cifras cercanas al $10 \%$,(Corzo Gómez et al., 2010, 2014).Este patrón, por su distribución especial puede predisponer a lesiones por extravasación del catéter durante la venopunción o a compromiso de estructuras aledañas, como las mencionadas por Cartwright (2004): flebitis mecánica, extravasación, hematoma o punción accidental de una arteria o nervio. Corzo Gómez et al. (2016) consideran la importancia de darle una aplicación clínica al concepto anatómico de los patrones venosos, en particular con el patrón tipo IV. De igual manera se debe resaltar la existencia de varias venas cefálicas, dos venas cefálicas entre el $13 \%$ y 13,5 \% y tres venas entre el 1 y $2 \%$ en el presente estudio.

Estos datos se suman cada vez más a la relación que se hace frente a comolas variaciones anatómicas en las venas pueden predisponer a complicaciones. Lirk et al. advirtieron de la existencia de una arteria radial accesoria cercana al sitio de canalización de la vena cefálica. del Sol \& Olave (1991) recomendaron que para la punción venosa de la fosa cubital se usara preferiblemente la vena intermedia basílica (VIB) o la vena intermedia del codo (VICo) por su cercanía con el nervio cutáneomedial del antebrazo.
Cárcoba Rubio \& Ceña Santorcuato (2010) sugieren para instalar un catéter venoso central de acceso periférico en la fosa antecubital, usar las venas basílica, mediana (intermedia del antebrazo) y cefálica, del lado derecho, en ese orden, puesto que la vena basílica del brazo derecho dado su acceso más directo la aurícula derecha.

Dentro de las recomendaciones que se hacen en las guías de Enfermería y manuales de procedimiento de las Instituciones de Salud se enfatiza en medidas como las mencionadas por Ochoa et al. (2005), las cuales incluyen localizar y seleccionar la vena a canalizarde acuerdo al propósito de la terapia intravenosa, palpándola con la yema de los dedos y evitar la utilización de aquellas que tengan las siguientes características: punción previa reciente, anormalidades anatómicas, rigidez, demasiada movilidad, inflamación o tromboflebitis.

El abordaje de una venopunción sin el conocimiento completo de la anatomía puede generar lesiones sobre estructuras vasculares y nerviosas adyacentes a las venas tal como lo señalaron Yamada et al. (2008). En este sentido, consideramos que es muy importante ahondar en el estudio de las variaciones anatómicas venosas superficiales del miembro superior, y mejorar los mecanismos de difusión de estos nuevos conceptos en el medio académico, previamente Corzo Gómez et al. (2016) llamaron la atención sobre la posible relación de los patrones venosos y las complicaciones durante la venopunciónen particular en poblaciones infantiles, y la necesidad de fomentar en las escuelas de Enfermería y Medicina la enseñanza de los patrones venosos y sus implicaciones de riesgo.

\section{AGRADECIMIENTOS}

Financiado por la Dirección Nacional de Investigaciones de la Universidad Cooperativa de Colombia. Proyecto 2728: «Vena cefálica que drena en la vena basílica como factor predisponente de lesión durante la venopunción periférica o durante la inserción periférica de un catéter venoso central».

CORZO, G. E. G.; CÁCERES, R. D. I.; CALDERÓN, M. R. \& LÓPEZ, R. L. A. Importance of the cephalic vein termination site. Int. J. Morphol., 39(3):705-709, 2021.

SUMMARY: The superficial veins of the ulnar fossa have been studied for various purposes, venipuncture, catheterization, fistulae, among others. Various venous patterns have been described in this region in different populations. In one of these, the cephalic vein ends in the basilic vein at the level of the forearm, generating 
possible risks of injury during cannulation. In the present study, 200 upper limbs of 100 Colombians, 50 women and 50 men, were evaluated, 70 of them born in the city of Bucaramanga and 30 in the city of Villavicencio. The number of cephalic veins and their termination site, on the arm or forearm, were recorded. The venous pattern that predominated in both upper limbs was type I. Pattern IV in which the cephalic vein ends in the basilic vein occurred in 12 cases $(6 \%)$ in the right upper limb and in 17 cases $(8.5 \%)$ in the left upper limb. Two cephalic veins were found between $13 \%$ and $13.5 \%$ depending on the side and three cephalic veins between $1 \%$ and $2 \%$. Knowing this particularity of venous patterns could reduce the risk of complications during venipuncture.

KEY WORDS: Cephalic vein; Venopuncture; Venous Patterns.

\section{REFERENCIAS BIBLIOGRÁFICAS}

AlBustami, F.; Altarawneh, I. \& Rababah, E. Pattern of superficial venous arrangement in the cubital fossa of adult Jordanians. Jordan Med. J, 48(4):269-74, 2014

Bekel, A. A.; Bekalu, A. B.; Moges, A. M. \& Gebretsadik, M. A. Anatomical variations of superficial veins pattern in cubital fossa among North West Ethiopians. Anat. J. Afr., 7(2):1238-43, 2018.

Boeson, M. B.; Hranchook, A. \& Stoller, J. Peripheral nerve injury from intravenous cannulation: a case report. AANA J., 68(1):53-7, 2000.

Cárcoba Rubio, N. \& Ceña Santorcuato, S. Cateterización venosa central de acceso periférico mediante técnica Seldinger modificada en la urgencia hospitalaria. Enferm. Glob., (20):1-8, 2010.

Cartwright, D. W. Central venous lines in neonates: a study of 2186 catheters. Arch. Dis. Child. Fetal Neonatal Ed., 89(6):F504-8, 2004.

Corzo Gómez, E. G.; Gómez Díaz, O. L.; Niño Mantilla, M. E.; Ramírez Vargas, L. M. \& Zárate Sierra, L. M. Distribution pattern of the veins of the cubital fossa in a sample of people born in the Department of Santander, Colombia. Int. J. Morphol., 32(1):221-6, 2014.

Corzo Gómez, E. G.; Gómez Díaz, O. L.; Niño Mantilla, M. E.; Rey Triana, R. J. \& Pedraza Díaz, L. J. Distribution Pattern of the veins of the cubital fossa in a sample of people born in Bucaramanga, Colombia. Int. J. Morphol., 28(4):1011-8, 2010.

Corzo Gómez, E. G.; Robles Carreño, M. I.; Díaz Díaz, A. M. \& Osma Zambrano, S. E. Relationship between surface forearm vein pattern and complications of peripheral and central venous catheterization in a sample of children treated in the pediatric intensive care unit of Santander Colombia. Int. J. Morphol., 34(3):885-9, 2016.

del Sol, M. \& Olave, E. Venas de la fosa cubital en el hombre. Sitios de punción. Rev. Chil. Cienc. Med. Biol., 1(1):49-53, 1991.

del Sol, M.; DeAngelis, M. A. \& Bolini, P. A. D. Formações venosas na fossa cubital da criança. Pediatr. Mod., 23(4):225-31, 1988.

del Sol, M.; Lagos Mardones, M. \& Torres Bustos, E. Venous formations in the cubital fossa of Mapuche: bioscopy study. Int. J. Morphol., 25(4):885-94, 2007.

Dharap, A. S. \& Shaharuddin, M. Y. Patterns of superficial veins of the cubital fossa in Malays. Med. J. Malaysia, 49(3):239-41, 1994.

Horowitz, S. H. Venipuncture-induced neuropathic pain: the clinical syndrome, with comparisons to experimental nerve injury models. Pain, 94(3):225-9, 2001.

Lirk, P.; Keller, C.; Colvin, J.; Colvin, H.; Rieder, J.; Maurer, H. \& Moriggl, B. Unintentional arterial puncture during cephalic vein cannulation: case report and anatomical study. Br. J. Anaesth., 92(5):740-2, 2004.
Ochoa, R. V.; Arroyo de Cordero, G.; Lee, G. M.; Jiménez Sánchez, J.; Galindo Barrera, M.; Hernández García, G.; Sánchez González, J. M.; Hernández Gamboa, L. E.; Campos Castolo, E. M.; Chávez Villanueva, M.; et al. Recomendaciones específicas para enfermería sobre el proceso de terapia endovenosa. Rev. Mex. Enferm. Cardiol., 13(1-2):53-60, 2005.

Racadio, J. M.; Doellman, D. A.; Johnson, N. D.; Bean, J. A. \& Jacobs, B. R. Pediatric peripherally inserted central catheters: complication rates related to catheter tip location. Pediatrics, 107(2):E28, 2001.

Ukoha, U. U.; Oranusi, C. K.; Okafor, J. I.; Ogugua, P. C. \& Obiaduo, A. O. Patterns of superficial venous arrangement in the cubital fossa of adult Nigerians. Niger. J. Clin. Pract., 16(1):104-9, 2013.

Vasuda, T. A study on superficial veins of upper limb. NJCA, 2:204-8, 2013.

Yamada, K.; Yamada, K.; Katsuda, I. \& Hida, T. Cubital fossa venipuncture sites based on anatomical variations and relationships of cutaneous veins and nerves. Clin. Anat., 21(4):307-13, 2008.

\section{Dirección para correspondencia: \\ Edgar Giovanni Corzo Gómez. \\ Carrera 24 número 35-191 \\ Altos de cañaveral sexta etapa Torre 3 apto 101 \\ COLOMBIA}

E-mail: Edgar.corzo@campusucc.edu.co

Recibido : 19-02-2021

Aceptado: 03-04-2021 